\title{
Micro-Raman Spectroscopy of Natural and Synthetic Ferritins and Their Mimetics
}

\author{
M. SzyBowicz ${ }^{a}$, M. Koralewski ${ }^{b, *}$, J. Karoñ $^{c}$ And L. Melnikova ${ }^{d}$ \\ ${ }^{a}$ Faculty of Technical Physics, Poznań University of Technology, Nieszawska13A, 60-965 Poznań, Poland \\ ${ }^{b}$ Faculty of Physics, A. Mickiewicz University, Umultowska 85, 61-614 Poznań, Poland \\ ${ }^{c}$ Clinic of General Surgery, Poznań University of Medical Sciences, Szwajcarska 3, 61-285 Poznań, Poland \\ ${ }^{d}$ Institute of Experimental Physics, SAS, Watsonova 47, 04001 Kosice, Slovakia
}

\begin{abstract}
Micro-Raman spectroscopy was used to studies of naturally occurring biogenic ferritin, synthetic ferritin with magnetic core (magnetoferritin) as well as their several mimetics. We demonstrate the ability of micro-Raman spectroscopy to discriminate between ferritin and magnetoferritin used in the studies. The results are promising for further use of the Raman spectroscopy as potential tool to distinguish between the forms of iron present in biogenic materials and biological tissues.
\end{abstract}

DOI: $10.12693 /$ APhysPolA.127.534

PACS: 87.15.-v, 87.64.Je

\section{Introduction}

Discovery of biological magnetite in the human brain [1] and recent studies indicated that neurodegenerative diseases [2] are closely associated with occurrence of iron $\mathrm{Fe}^{2+}$. The structure, quality and quantity of magnetic structures in the brain have not been fully determined yet and it has not been established if they are related to the origin of the neurodegenerative diseases or to their consequences $[3,4]$. Ferritin is a protein which serves as a storage capsule of iron among humans also inside animals, plants and microbial organisms. The protein itself (apoferritin) is an almost perfect sphere, with outer diameter about $12 \mathrm{~nm}$, hollow inside, with 7 to $8 \mathrm{~nm}$ inner diameter. The central core of ferritin consists of iron(III) oxyhydroxide known as ferrihydrite. Mann and co-workers [5] showed that ferritin can be used as a bioreactor of nanometer size for producing monodisperse metal or metal oxide particles. Especially magnetic iron oxide was synthesised inside ferritin to produce so-called magnetoferritin. Of particular interest is the search for methods allowing detection and discrimination of magnetic material inside ferritin proteins both in vitro and in vivo. Very recently we showed that combined magnetooptical methods are very useful to discriminate between ferritin and magnetoferritin $[6,7]$. The use of the Raman spectroscopy (RS) for the detection and diagnosis in biomedicine is a growing research field, however mostly related to oncology (e.g. $[8,9])$. It is known that nonheme iron is present in human body in a wide variety of forms including nanoscale particles. In this paper we propose to use RS as a potential tool for future diagnosis of pathology related to naturally occurred nanoscale iron biominerals and compounds which are present in human

* corresponding author; e-mail: koral@amu.edu.pl organs. The present paper is devoted to the comparative study of the Raman spectra of natural and synthetic ferritins and their mimetics.

\section{Experiment}

As naturally occurring biogenic ferritin - horse spleen ferritin (HSF) obtained from Sigma were used. Synthetic ferritin with magnetic core (magnetoferritin) were obtained by using apoferritin (Sigma) and suitable chemical synthesis procedures $[10,11]$. As model ferrihydritelike mineral parenteral iron formulation (Ferrumlek, Dexferrum, CosmoFer) and iron-dextran complex commercially obtainable from Sigma (Fe-dextran $(\mathrm{S})$ ) were used. As nanoscale magnetite was used commercially available suspension of iron oxide nanoparticles coated by dextran known as Endorem (Guerbet, France) which is clinically used as MRI contrast agent and was characterized in our paper [12]. The Raman scattering spectra of nanoparticles were investigated in the spectral range of $150-1000 \mathrm{~cm}^{-1}$ at room temperature. The nonpolarized Raman spectra were recorded in the backscattering geometry using inVia Renishaw micro-Raman system. As an excitation light we used near infrared line of solid state laser operating at $785 \mathrm{~nm}$. The laser beam was tightly focused on the sample surface through a Leica $50 \times$ LWD microscope objective (LWD - long working distance) with numerical aperture (NA) equal to 0.5 leading to a laser beam diameter about $2 \mu \mathrm{m}$. To prevent any damages of the sample an excitation power was fixed at $1 \mathrm{~mW}$. The inVia Raman spectrometer allowed for recording the Raman spectra with the spatial resolution of about $1 \mu \mathrm{m}$. The spectral resolution was $1 \mathrm{~cm}^{-1}$.

\section{Results and discussion}

The details about iron content, size and type of mineral of the core of studied mimetics may be found in our recent papers $[12,13]$. Briefly XRD patterns of mother liquors give information about akaganeite $(\beta-\mathrm{FeOOH})$ and magnetite present in the core of all parenteral formulation 
used and Endorem, respectively. The Raman spectra of iron oxides and oxyhydroxides, HSF and low loaded magnetoferritin (LLM) are shown in Figs. 1-3. We have registered spectra of akaganeite nanocrystals using the iron-dextran complex (Fe-dextran $(\mathrm{S})$, iron concentration in mother liquor $\left.-c^{\mathrm{Fe}}=100 \mathrm{~g} / \mathrm{L}\right)$ and the following iron formulation: Fe-polymaltose (Ferrumlek $-c^{\mathrm{Fe}}=$ $50 \mathrm{~g} / \mathrm{L}$ ), Fe-dextran (AR) (Dexferrum $-c^{\mathrm{Fe}}=50 \mathrm{~g} / \mathrm{L}$ ), $\mathrm{Fe}-$ dextran $(\mathrm{Ph})\left(\mathrm{CosmoFer}-c^{\mathrm{Fe}}=50 \mathrm{~g} / \mathrm{L}\right)$.

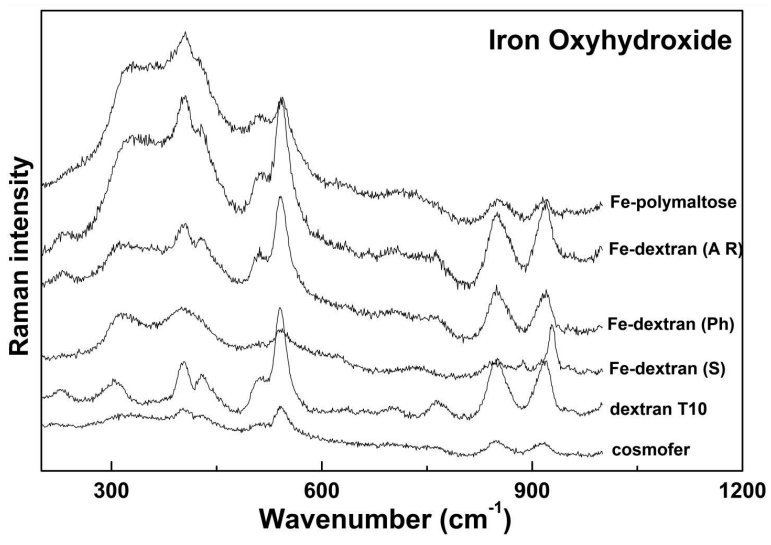

Fig. 1. Raman spectra of akaganeite nanocrystals of different iron formulation $(\beta-\mathrm{FeOOH})$, iron - dextran from Sigma and pure dextran (Dextran T10) as well as lyophilised CosmoFer (cosmofer).

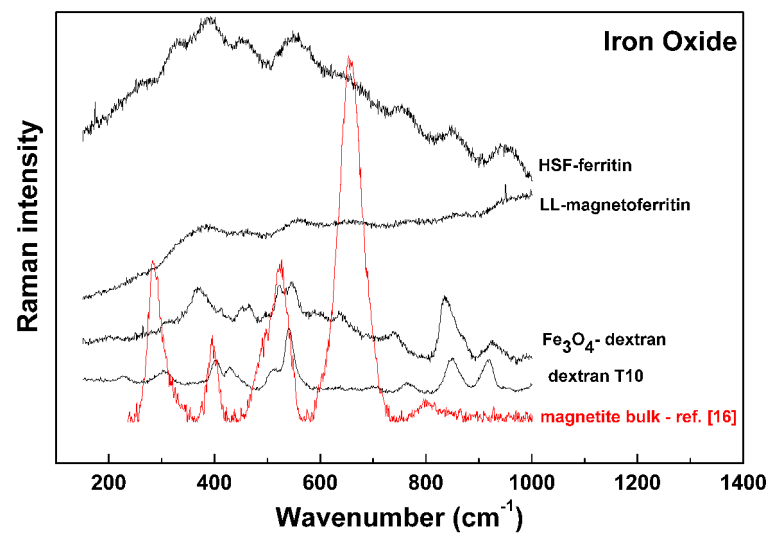

Fig. 2. Raman spectra of bulk magnetite, nanoscale magnetite, LL-magnetoferritin and HSF as well as pure dextran.

For comparison RS of pure dextran T10 (powder) and lyophilised form of CosmoFer (cosmofer) is also enclosed in Fig. 1. It has been known from the literature that for typical $\beta$-FeOOH oxyhydroxide the observed Raman bands are at the wavenumber position: 310, 385, 480, $535,615,675,725 \mathrm{~cm}^{-1}[14,15]$. As seen in Fig. 1 the observed Raman bands after elimination of vibrations related to dextran are at the positions: $-307(\mathrm{Fe}-\mathrm{O}$ symmetric bending), 385 ( $\mathrm{Fe}-\mathrm{O}-\mathrm{Fe}$ symmetric stretching), 533, 619, $725 \mathrm{~cm}^{-1}$ (Fe-OH asymmetric stretching) which are well correlated with $\beta$-FeOOH. Additionally, some small differences between analysed iron formulation may be noticed. It needs to be stressed that vibrational properties of nanoparticles strongly depend on their morphology. It needs to be noted that for lyophilised sample vibrations related to polysaccharide can be relatively damped but this procedure does not help much in better resolving the RS of studied compounds.
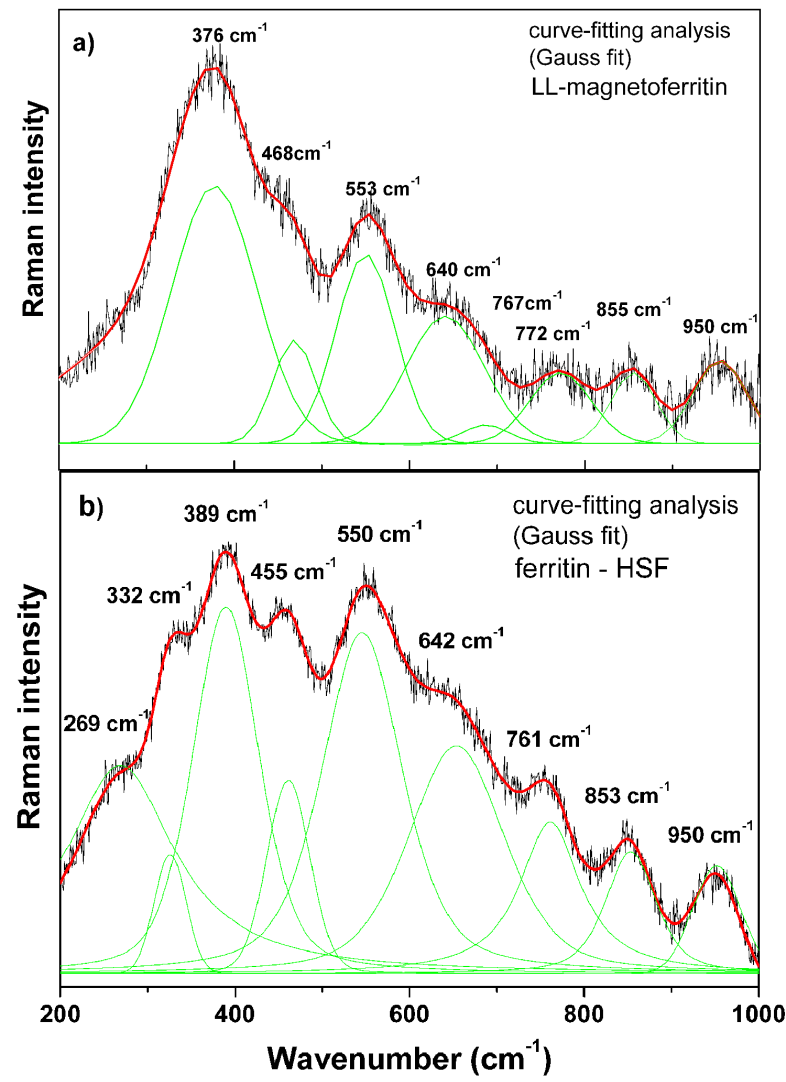

Fig. 3. Room temperature Raman spectra deconvolution of LL-magnetoferritin - a) and HSF - b).

In Fig. 2 we present comparison of Raman scattering spectra of bulk magnetite [16], nanoscale magnetite $\left(c^{\mathrm{Fe}}=11 \mathrm{~g} / \mathrm{L}\right), \operatorname{LLM}\left(c^{\mathrm{Fe}}=0.126 \mathrm{~g} / \mathrm{L}\right)$ and $\mathrm{HSF}$ $\left(c^{\mathrm{Fe}}=7.1 \mathrm{~g} / \mathrm{L}\right)$ as well as pure dextran. Magnetite has a spinel structure with five Raman bands: three $T_{2 g}$ symmetry, one $E_{g}$ symmetry and one $A_{1 g}$ symmetry. Maghemite has also a spinel structure and can be seen as an iron-deficient form of magnetite.

In the spectra of LLM, we can identify bands appearing about $376 \mathrm{~cm}^{-1}\left(E_{g}, \mathrm{Fe}-\mathrm{O}\right.$ symmetric stretching), $468 \mathrm{~cm}^{-1}\left(T_{2 g}, \mathrm{Fe}-\mathrm{O}\right.$ asymmetric bending $), 640 \mathrm{~cm}^{-1}$ $\left(A_{1 g}, \mathrm{Fe}-\mathrm{O}\right.$ symmetric stretching) connected with $\gamma$ $\mathrm{Fe}_{2} \mathrm{O}_{3}$ phase (see Table). It may be noticed that for this particular magnetoferritin no bands related to magnetite may be observed (see Table) in agreement with magnetooptical results obtained for the same compounds [7]. In the case of the ferritin we recorded bands about 269 , $332,389,455,550,642,761,853$ and $950 \mathrm{~cm}^{-1}$. Two 
bands about 550 and $642 \mathrm{~cm}^{-1}$ are close to bands which are also found in LL-magnetoferritin which means that ferrihydrite-like material may be also present in the LLM core or some cores may have ferrihydrite-like mineral. The Raman bands for LLM and HSF are relatively very weak which is related to much lower Fe amount in comparison to Endorem or akaganeite type iron formulation. A good quality of the Raman spectra depends also on the laser power used. However, too strong laser beam may cause phase transition from one to another iron oxide structure. Good balance should be found in each particular experiments performed in the future especially when using $\mu$-Raman.

\section{TABLE}

Gaussian shaped-Raman modes obtained from deconvolution analysis of the Raman scattering for natural and synthetic ferritins and their mimetics and pure dextran with literature date for some iron oxides.

\begin{tabular}{l|l}
\hline \hline Compound & Raman wavenumber $\left[\mathrm{cm}^{-1}\right]$ \\
\hline Magnetite [16] & $283,400,491,526,650,687,798$ \\
Maghemite [17, 18] & $365,376,472,511,640,700$ \\
Magnetoferritin & $376,468,553,640,767,772,855$, \\
- LLM & 950 \\
Ferritin - HSF & $269,332,389,455,550,642,761$, \\
& 853,950 \\
$\mathrm{Fe}_{3} \mathrm{O}_{4}$ - dextran & $307,368,415,455,465,495,525$, \\
- Endorem & $545,584,645,738,835,922$ \\
Akaganeite & $307,331,385,456,533,558,619$, \\
- iron formulation & $725,849,950$ \\
Dextran T10 & $228,303,403,433,509,540,703$, \\
& $767,850,916$
\end{tabular}

In Fig. 3 we present deconvolution of the Raman spectra of LLM and HSF which helps in better resolving contributions related to particular vibrations. In all studied cases we used Gaussian curve fitting method to obtain Raman bands from the RS of ferritins and their mimetics compounds. The results were collected in Table together with some literature data for iron oxides. As seen from Fig. 3 and Table there are several bands below $700 \mathrm{~cm}^{-1}$ which are quite at different positions allowing to discriminate between LLM and HSF.

\section{Conclusions}

All compounds studied show Raman spectra related to $\mathrm{Fe}-\mathrm{O}$ frequency (different minerals may be discriminated) however they are strongly masked by protein or polysaccharide bands. Thus, deconvolution of obtained spectra allows for more precise interpretation of the Raman spectrum of particular studied compounds (see Fig. 3 and Table). From presented studies it can be concluded that ferritin and magnetoferritin Raman spectra show different features which is promising for discrimination between them in human tissues or fluids. In general, this method may be potentially useful to observe change in the structure due to the increase of the temperature or to observe coexistence of different iron oxide phases in the studied material. Characterization of these iron oxides phases in human tissues or fluids needs further studies.

\section{Acknowledgments}

We gratefully acknowledge American Regent Inc. (Shirley, New York, USA) and Pharmacosmos (Denmark) for providing Dexferrum and dextran, respectively. The work was partialy supported by the project Vega 0045 and APVV 0171-10.

\section{References}

[1] J.L. Kirschvink, A. Kobayashi-Kirschvink, B.J. Woodford, Proc. Natl. Acad. Sci. 89, 7683 (1992).

[2] C. Quintana, Mini-Rev. Med. Chem. 7, 961 (2007).

[3] J. Galazka-Friedman, Hyperfine Interact. 182, 31 (2008).

[4] J. Galazka-Friedman, A. Friedman, E.R. Bauminger, Hyperfine Interact. 189, 31 (2009).

[5] F.C. Meldrum, B.R. Heywood, S. Mann, Science 257, 522 (1992).

[6] M. Koralewski, M. Pochylski, Z. Mitróová, M. Timko, P. Kopčanský, L. Melníková, J. Magn. Magn. Mater. 323, 2413 (2011).

[7] M. Koralewski, J.W. Kłos, M. Baranowski Z. Mitróová, P. Kopčanský, L. Melníková, M. Okuda, W. Schwarzacher, Nanotechnology 23, 355704 (2012).

[8] T. Meyer, N. Bergner, C. Bielecki, C. Krafft, D. Akimov, B.F.M. Romeike, R. Reichart, R. Kalff, B. Dietzek, J. Popp, J. Biomed. Opt. 16, 021113 (2011).

[9] B. Brożek-Płuska, I. Placek, K. Kurczewski, Z. Morawiec, M. Tazbir, M. Abramczyk, BJ. Mol. Liq. 141, 145 (2008).

[10] Z. Mitróová, L. Melníková, J. Kováč, M. Timko, P. Kopčanský, Acta Phys. Pol. A 121, 1318 (2012).

[11] L. Melníková, Z. Mitróová, M. Timko, J. Kovac, M. Koralewski, M. Pochylski, M.V. Avdeev, V.I. Petrenko, V.M. Garamus, L. Almasy, P. Kopčanský, Magnetohydrodynamics 49, 293 (2013).

[12] A. Skumiel, A. Szlaferek, W. Kowalski, M. Koralewski, Pol. J. Environ. Stud. 1, (90 (2010)).

[13] M. Koralewski, M. Pochylski, J. Gierszewski, J Nanopart. Res. 15, 1902 (2013).

[14] N. Boucherit, P. Delicher, S. Joiret, A. Hugot-Le Goff, Mater. Sci. Forum 44-45, 51 (1989).

[15] T. Ohtsuka, Mater. Trans. JIM 37, 67 (1996).

[16] T. Laetsch, R.T. Downs, Software for Identification and Refinement of Cell Parameters from Powder Diffraction Data of Minerals Using the RRUFF Project and American Mineralogist Crystal Structure Databases, in: Abstracts from the 19th General Meeting of the International Mineralogical Association, Kobe (Japan), 2006.

[17] A.M. Jubb, H.C. Allen, ACS Appl. Mater. Interfaces 2, 2804 (2010).

[18] T. Ohtsuka, K. Kubo, N. Sato, Corrosion 42, 476 (1986). 